\section{Therapeutic Alliance and Adherence in Family Therapy}

\section{La alianza y la adherencia terapéuticas en la terapia familiar}

\section{Ismael Fernando Díaz Oropeza ${ }^{1}$}

1 Universidad de las Américas, A.C., México.

\section{Correspondence about this article should be addressed to:}

Ismael Fernando Díaz Oropeza, Departamento de Ciencias del Comportamiento y del Desarrollo, Universidad de las Américas, A.C., Puebla 223, Col. Roma, Distrito Federal, México, C.P. 06700, E-mail: idiaz@udladf.mx

\begin{abstract}
The purpose of this study was to test out the alliance and treatment adherence as explanatory factors that contribute to the effectiveness of family therapy. Seven family therapists and four clients participated voluntarily. A system for observing family therapy alliances (SOFTA-o) and self-reports (SOFTA-s) were used to measure therapeutic alliance. Therapeutic adherence was measured using a Family Therapy Adherence Scale and Goal Attainment Scale to measure outcome. Third and fifth therapy sessions were recorded and scales were applied at the end of these sessions. Findings indicated a low relationship between clients' perception of the therapeutic alliance and therapeutic adherence, a strong relationship between therapeutic alliance, adherence and outcome was found. Clinical, training and research implications are discussed.

Keywords: Therapeutic alliance, adherence, outcome, family therapy.
\end{abstract}

\section{Resumen}

El propósito de este estudio fue probar que la alianza y la adherencia al tratamiento son factores explicativos que contribuyen a la efectividad de la terapia familiar. Participaron voluntariamente siete terapeutas familiares y cuatro clientes. Se utilizó el sistema de observación de la alianza terapéutica en la intervención familiar (SOATIF-0) y los autorreportes (SOATIF-s) para medir la alianza terapéutica. La adherencia terapéutica se midió mediante la escala de adherencia a la terapia familiar y la escala de logro de metas para medir los resultados. Se videograbaron la tercera y quinta sesiones de terapia, las escalas se aplicaron al final de estas mismas sesiones. Los hallazgos indicaron una baja relación entre la percepción del cliente de la alianza terapéutica y la adherencia. Se encontró una fuerte relación entre la alianza terapéutica, la adherencia y los resultados. Se discuten las implicaciones para la práctica clínica, el entrenamiento y la investigación. Palabras clave: Alianza terapéutica, adherencia, resultados, terapia familiar. 
It is well known that the quality of the relationship between client and therapist is an essential part of therapeutic success. However, most of family therapy approaches have not incorporated this construct to theoretical conceptualization and clinical models. The majority of clinical supervisors think that therapist's relational skills are more important than techniques or their theoretical orientation (Blow \& Sprenkle, 2001). Bordin (1979) suggested a pantheoretical definition of therapeutic alliance as fit and collaboration among clients and therapists, identifying three components: a) agreement with goals, b) agreement with tasks and activities and, c) positive bond. Bordin's theory is based in four assumptions: 1) all forms of psychotherapy require an alliance, 2) the difference is alliance type for each therapy (individual, family or couple), 3) therapy effectiveness is due to alliance' strength and, 4) alliance' strength is determined by particular characteristics of clients and therapists (Johnson \& Wright, 2002).

Widely it is accepted that therapeutic alliance is an interpersonal and intrapersonal phenomena (Horvath, 2006). Based on interpersonal aspect, Pinsof (1995) defined therapeutic alliance in family therapy as clinically relevant part of relationship among therapist and system clients that includes relationship within therapeutic system and, therapists' and clients' ability to collaborate mutually with tasks and therapeutic goals. He describes content dimension that involves original Bordin's conceptualization and interpersonal dimension is subdivided into: individual, subsystem, whole system and within system.

The complexity of alliance components and dimensions, involves multiple levels in family therapy (Johnson \& Ketring, 2006). Family therapists have to maintain multiple alliances simultaneously, which represents greater demands to therapist (Beck, Friedlander \& Escudero, 2006). Typically, a family therapy session involves circular interactions among individuals, that is the 'expanded therapeutic alliance' (Escudero, Friedlander, Varela \& Abascal, 2008), which is an unique factor to conjoint family and couple therapy (Sprenkle \& Blow, 2004). When an adequate therapeutic alliance is constructed, family members collaborate to improve family relationship, to reach therapeutic goals and developing a unit sense within the family related to the therapy (Beck, Friedlander \& Escudero, 2006).

The therapeutic alliance has had a positive effect in systemic family therapy (Dinger, Strack, Leischsenring \& Schauenberg, 2007). It exists a few doubts that alliance between client and therapist is consistently related to therapy outcomes (Johnson \& Ketring, 2006) and, it is a strong predictor of progress in marital therapy (Knobloch, Pinsof \& Mann, 2004). In marital and family therapy, therapeutic alliance provides a context for interpersonal relationship and, it offers an opportunity for a corrective experience, that it could be considered as a necessary condition for therapeutic change (Rait, 2000).

It seems that alliance in family therapy is fluid, it is fortified during therapeutic process, it has a direction and it grows in importance throughout the time (Friedlander, Escudero \& Heatherington, 2006). During the initial stages of therapy, it is crucial to create a security space, involving family members and identifying some aspects on which all agree (Pinsof, 1995). In first and third sessions a pattern of linear increased is produced (Stevens, Muran, Safran, Gorman,Winston, 2007).

Although traditionally the centrality of the alliance has been emphasized, it is a necessary but not a sufficient condition for effectiveness of family therapy. The other main ingredient is therapeutic adherence, which has received little attention in marital and family therapy literature.

It exists an enormous conceptual divergence because diverse expressions have been used to talk about the therapeutic adherence, which has generated confusion and a lack of consensus among professionals and researchers (Martin, 2004). In psychological literature, the terms compliance and adherence, are used indifferently. Compliance has been criticized, because it involves an authoritarian and coercive role of medical team and, a passive role is assigned to the client, ignoring the client's contribution to the process.

In order to surpass these limitations, the term therapeutic adherence is suggested, to express the interactive character of relationship between the client and the therapist. Di Mateo y Di Nicola (1985) conceive adherence as a commitment, an active implication, a voluntary collaboration of client in a course of treatment in mutual agreement, in order to produce therapeutic outcomes. In this conceptualization, clients are recognized as an active agents and requires he/she carries out concrete actions for the advance of the treatment (Basterra, 1999). Adherence and alliance are relational aspects and, pantheoretical concepts that could explain therapeutic outcomes. 
Due to the relevance that alliance and adherence have within the therapeutic process, this study tries to relate these two components and to propose them like explanatory factors that contribute to the obtaining of wished therapeutic outcomes. The purpose of this research was to test out the alliance and treatment adherence are explanatory factors that contribute to the effectiveness of family therapy. The hypothesis were:

$\mathrm{H}_{1}$ : It exists a relationship between therapeutic alliance and adherence since clients' perspective.

$\mathrm{H}_{2}$ : It exists a relationship between therapeutic alliance and effectiveness.

$\mathrm{H}_{3}$ : It exits a relationship between adherence and effectiveness.

\section{Method}

Participants

Seven family therapists participated voluntarily, they were psychologists with a masters' degree. Three were woman and 4 men. Six therapists have between 3 to 7 years of experience and 1 has 8 to 12 years of clinical experience at the time of the study.

Four families participated voluntarily and, they were not attending another therapy in parallel form. They were integrated as it follows:

Family $A$. The client is an organizational psychologist of 25 years of age, she attended the therapy to find the form to finish with relationship patterns that she considered harmless, like: violence, aggression and infidelity. Her father had heart attack 3 months ago and her mother acts like her nursemaid. She has two older brothers, that became independent recently. His older brother abused her when she was young. At the moment, the client lives single. He has had 5 sessions in therapy.

Family $B$. The client is 29 years old and she is a designer, she went to the therapy because she had marital problems and wanted to prevent a depression. She describes her husband as not affectionate, distant and fulfills his role of supplier solely. They have frequent fights and she is worried about her son being affected by this situation. She has had 5 sessions in therapy.

Family C. The client is a 24 years old woman. She has a 5 years old daughter. She attended to therapy because she had problems with her daughter's discipline and misbehavior. The mother describes her as uncontrollable, arrogant and, crude. There are no limits at home, inconsistency in punishments and prizes, in addition that her husband disconfirms her in front of her daughter. They have had 5 sessions in therapy.

Family $D$. The clients are a couple integrated by a 38 years old woman and a 37 years old man. They went to therapy because they wanted to grow like individuals and family, to fix their marriage and to be a functional family. They have a 9 and 4 years old daughters and a 9 months old baby. They have a file of mistreat, verbal and psychological violence and infidelity from both. They have had 5 sessions in therapy.

\section{Instruments}

System for observing family therapy alliances (SOFTA-o) (Friedlander, Escudero and Heatherington, 2006), which is an observational rating scale based on the behavioral expressions of therapeutic alliance of the clients, as well as in the observable contributions of the therapist to the alliance. The score varies from very strong of +3 to -3 very problematic one. It includes a set of behavioral indicators that represent positive and negative expressions of each four dimensions that compose SOFTA model: 1) Engagement in the Therapeutic Process, 2) Emotional Connection with the Therapist, 3) Safety within the Therapeutic System, and 4) Shared Sense of Purpose within the Family.

SOFTA self-reports (SOFTA-s) (Friedlander \& Escudero, 2002). It is a questionnaire of 16 items with four scales, corresponding to the four dimensions of the SOFTA. There are two versions: for the client and the therapist.

Family Therapy Adherence Scale (Díaz, 2010). It is an instrument that consists of 13 items that were administered by the therapist to the clients. This is a Likert type scale, which scores vary from 1 , strongly disagree to 5 , strongly agree. It consists of 3 dimensions: 1) treatment compliance, 2) involvement of client and, 3) therapist-client collaboration.

Goal Attainment Scaling (Kiresuk, Smith \& Cardillo, 1994). It is a tool designed to be used in clinical situations where specific goals are set jointly between the therapist and client as part of a case management process. Therapists and clients identify the specific goal(s), to define what the expected outcome would 
be at the end of the process. The score rating varies from +2 much more than expected to -2 much less than expected.

\section{Procedures}

Family therapists were contacted by means of a letterinvitation. The purpose was to explain the goals of the study and to schedule an appointment in order to extend the information about the project. All family therapists and clients signed an informed consent that includes the authorization to participate in the study and to record the sessions.

Third and fifth sessions were recorded in video, because it is during this period when the alliance increases (Stevens, Wall, Safran, Groman \& Winston, 2007). In addition, at the end of these sessions, both, the family and therapist completed the SOFTA selfreports and clients Family Therapy Adherence Scale. In order to develop the protocol for the scale of profit of goals (Goal Attainment Scaling), the researcher consulted the therapist to identify the significant goals for each member individually, subsystem or complete family using a scale of potential behavioral changes.

\section{Results}

Contextual variables of family therapists

The group of therapists was formed by 4 men and 3 women. Six therapists have of 3 to 7 years of experience and 1 had 8 to 12 years of clinical experience at the time of the study. Four therapists described their training like the emphasis in the development of their own therapeutic style and, three of them considered it like the emphasis in the development of clinical abilities.

Four therapists considered their work as a collaborative practice, 2 narrative and 1 solutionbuilding.

\section{Contextual variables of clients}

Four families integrated by 5 individuals participated voluntarily in this study: 3 women and 2 men, age ranged from 24 to 38 years, with an average of 30.6 years, $S D=6.58$. Clients had $71.4 \%$ of attendance to the therapy sessions and $28,6 \%$ of unattendance.

Reliability

SOFTA self-reports. The version of the therapist had $\alpha=.89$ and the version of the client $\alpha=.79$
Family Therapy Adherence Scale. The version of the client presented $\alpha=.92$. The test-retest method applied to the instrument showed $r=.77, p=.124$

Relationship between therapeutic alliance and adherence in clients

The therapeutic alliance decreased while the adherence was relatively stable. At 3rd session, therapeutic alliance remained higher than the adherence, remaining parallel. In 5th session the alliance and treatment adherence intersected.

The therapeutic alliance as perceived by therapists and clients in the $3 \mathrm{rd}$ and 5 th sessions were measured by SOATIF-s. In Table 1 therapists and clients scores are presented. It shows that both presented a greater level of alliance in 3rd session, there were few differences between the alliance dimensions in the case of therapists and clients.

For therapists "engagement in the therapeutic process" dimension was more important, and for clients "emotional connection" and "safety within the therapeutic system" dimensions were more relevant. Regarding adherence, "involvement of client" dimension was the most important, as shown in table 2.

$\mathrm{H}_{1}$ : There was a low and directly proportional relationship between the alliance and treatment adherence in clients $\left(r_{s}=0.38\right.$, with five cases the level of significance of $p=.278$ ), thus accepting the null hypothesis there is no linear relationship between the alliance and adherence.

Relationship between therapeutic alliance and effectiveness

$\mathrm{H}_{2}$ : There was a significant relationship, moderate and directly proportional between the alliance and therapy effectiveness from the point of view of clients $(\eta=.76$, with 5 cases, $p=0.00$ ), therefore the null hypothesis is rejected if there is linear relationship between the alliance and therapy effectiveness.

\section{Relationship between adherence and effectiveness}

H3: There was a significant relationship, moderate and directly proportional between adherence and effectiveness of therapy from the perspective of clients $(\eta=.68$, with five cases, $p=0.00)$, therefore the null hypothesis is rejected if there is a linear relationship between adherence and effectiveness of therapy. 
Table 1. Scores of therapeutic alliance.

\begin{tabular}{|c|c|c|c|c|c|c|c|c|c|}
\hline \multicolumn{10}{|c|}{ Therapeutic alliance } \\
\hline & \multicolumn{4}{|c|}{ Therapists } & \multicolumn{5}{|c|}{ Clients } \\
\hline Session & Engagement & Connection & Safety & Purpose & Session & Engagement & Connection & Safety & Purpose \\
\hline $3 r d$ & 17.57 & 15.14 & 16.71 & 15.71 & $3 r d$ & 17.80 & 18.20 & 18.80 & 17.80 \\
\hline 5 th & 15.0 & 14.71 & 14.14 & 14.79 & 5 th & 15.40 & 14.80 & 12.40 & 14.80 \\
\hline$n$ & 7 & 7 & 7 & 7 & $n$ & 5 & 5 & 5 & 5 \\
\hline
\end{tabular}

Note: The means of total scores for each of the dimensions of SOATIF-s' therapist and client versions are showed.

Therapeutic alliance from therapist perspective

Therapists 1 and 2 .

The therapists 1 and 2 were working in cotherapy with the family $A$ had a light a light $(+1)$ and quite strong (+2) alliance in all dimensions. Therapists 1 and 2 showed uniform pattern, except in the dimension of engagement, where in the perspective of one observer, the therapist was more involved in the 3rd session as the therapist 2 . In the rest of the dimensions a similar pattern is seen in the establishment of the alliance.

Therapists 3 and 4.

The uniform pattern was observed in therapist 3 and 4 working in cotherapy with the family $B$. The alliance was slight (+1) and the size effect was not remarkable (0) due to a single client who participated in the sessions evaluated.

Therapist 5.

The therapist 5 who worked with the family $C$ had a very strong alliance $(+3)$ in the $3 r d$ session on engagement dimension to the mother and daughter. In the other dimensions, the alliance was quite strong $(+2)$ and stable throughout the sessions.

Therapists 6 and 7 .

The therapists 6 and 7 , who worked in cotherapy with the family $D$, had a very strong $(+3)$ and quite strong (+2) alliance, respectively, in the engagement dimension.

Table 2. Descriptives of dimension of Family Therapy Adherence

Scale.

\begin{tabular}{llll}
\hline Session & Compliance & Involvement & Collaboration \\
\hline 3rd & 17.80 & $\mathbf{2 2 . 8 0}$ & 18.40 \\
5th & 19.00 & $\mathbf{2 0 . 8 0}$ & 18.60 \\
$n$ & 4 & 4 & 4 \\
\hline
\end{tabular}

Note: The means of total scores for each of the dimensions of client version are showed.
Therapeutic alliance from client perspective

Family A.

The client presented the most consistent scores observed with SOATIF-o of the alliance on engagement and purpose dimensions, in which the alliance was slight $(+1)$. The connection and safety dimensions showed a fluctuation in the 5th and 3rd session.

Family B.

This client presented a light alliance $(+1)$ in the dimensions of connection and safety, quite strong $(+2)$ on the engagement and not remarkable (0) in the scale of shared sense of purpose.

Family $\mathrm{C}$.

The client made a quite strong alliance $(+2)$ in the dimensions of engagement, safety and purpose. But she had a light alliance $(+1)$ in the connection dimension.

Family D.

Clients had an different level of alliance, light $(+1)$ and a quite strong $(+2)$, with a predominance in the safety dimension.

Results of therapy outcomes

The goals that the therapist identified for each family, were altogether constructed between the therapist and each one of the clients. The scores of the scale were located by the therapist at the time and raged from -2 to +2 . The results show that generally, families $A$ and $B$ presented moderately positive results, family $C$ had moderately positive results in a goal and excellent results in the other and family $D$ had excellent results.

\section{Clinical improvement.}

Family A. The goal that defined the client was labeled as "healthy patterns of couple relationships", moderately had a positive result with +1 , which reflects an attainment level "a little more than 
expected" in the protocol of construction of the scale. The client reported a significant improvement in the establishment of relationships with her family and she declared to know how to choose a potential couple with clarity. The client attended 5 sessions, after which she left the process due to a business trip.

Family B. After 5 sessions, there was a mutual agreement between client and therapist to finalize the process. The client manifested important advances in terms of decision making in her marriage to settle down agreements with her husband and remarkably improved communication.

Family C. The client and her therapist defined two goals: "to learn to establish boundaries and rules" and "to spend more time with my daughter". The first goal had excellent results, located as an attainment level of "much more than expected". She evaluated it in this way because she has been surprised of her capacity to stablish clear boundaries to her daughter and, she has seen changes in her own and in her daugher's behavior. With respect to second goal, the score was +1 , which corresponds to a profit level of "a little more than expected", because she spent more quality time with her daughter. After 5 sessions, client and therapist reviewed their initial goals, decided to settle down a new therapeutic contract included 5 more sessions.

Family D. The clients defined goals as: "to improve my relationship with my partner, to learn to be a functional family" and "to have growth and to fix my marriage". With the facilitation of the therapist, they negotiated a scale of improvement of couple relationship. The attainment level was "excellent results" by the clients and the therapists, due to the advances that couple has had: fights and verbal agression stopped, they began to take agreements and they learn to negotiate. After 5 sessions, the process was finished by mutual agreement between the clients and the therapists.

\section{Discussion.}

These findings indicate a low relationship between clients' perception of the therapeutic alliance and therapeutic adherence. Specifically, data suggests that clients give great importance to the safety dimension in the therapeutic system and emotional connection. Additionally, clients give more importance to the involvement dimension in therapeutic adherence. For therapists, it is of great importance to engage in the therapeutic process.

These differences in alliance's dimensions between clients and therapists can be originated because of differences in expectations, roles and motivation of each one. For therapists it seems to be important to generate an engagement to the process, whereas for clients it is important to see as part of a treatment, being involved in therapy, to take the process seriously and, that change is possible. For clients emotional connection is more important, seeing the therapist as one important person in her/his life, that relationship is based on confidence, affection and interest, and that the client perceives that therapist is avalaible and therapist's experience is relevant. It is also important to clients to feel safe and comfortable within the therapeutic system.

Additionnally, an explanation to the alliances variation is that in the third session a linear increase of the alliance takes place (Stevens, Muran, Safran, Gorman,Winston, 2007), whereas in fifth session, the process termination took place; clients and therapists were more trims in the evaluation of the goals and the definition of a closing.

Like in the studies on individual therapy, correlation between the measures of the alliance intersessions was not found in the client as much as the therapist. Therapeutic alliance depends on characteristics of clients and therapists (Johnson \& Wright, 2002), as well as alliance's perception is dynamic and changes according to a particular event of a single session. In this sense, it would seem that each session is unique and different, but at the same time is related to the others. Therapeutic alliance is a very complex phenomena that involves multiple levels of interaction between participants within a session and between sessions (Johnson \& Ketring, 2006). On the other hand, findings indicate a significant relationship between therapeutic alliance and therapy outcomes, between adherence and therapy outcomes. The relationship between therapeutic alliance and outcome is well established in marital and family therapy (Dinger, Strack, Leischsenring \& Schauenberg, 2007; Johnson \& Ketring, 2006; Knobloch, Pinsof \& Mann, 2004; Pinsof, 2005). Family therapy involves an active and evolving relationship that may have a particular quality and contribution to therapeutic change (Rait, 2000). But, relationship between therapeutic adherence and outcome is little explored and more research is required to support the findings in this study. 
The following implications must be viewed tentatively.

\section{Clinical implications}

The implications for clinical practice related to the mental health professionals include that they seriously consider the idea of building strong therapeutic alliances with clients, as soon as possible during the initial phases of therapy and managed to keep it until the end (Pinsof, 1995). Clinicians must be aware of the possibility of ruptures or splits in the alliance, also should be alert to signs that suggest avoiding or correcting problems when they arise.

The establishment of a strong alliance with clients is crucial for the development of a process and satisfactory results, as it has been demonstrated by empirical evidence and clinical experience. The challenge is to build alliances that are perceived both by clients and by therapists working regardless of the model.

Regarding adherence, therapists must develop a deep understanding of the differences in the perceptions of clients about the adherence. The therapist would have to see his client as an active agent in the process, which is involved in therapy and is part of a program of treatment. The therapist would have to see his client as an ally that must be included in all decisions, actions and activities during the process.

\section{Training implications}

Training programs might assist trainees in understanding the influence of therapeutic alliance and adherence on outcomes. Based on this understanding, trainees must develop therapy skills in order to establish an adequate relationship with clients and involving them across the therapeutic process. Training process must emphasize the development of relational abilities, competitions to prevent and to repair split alliances.

Competency-based training for the establishment of an alliance and strong adherence involve an analysis of the competences and social skills of the therapiest in traning. The program would have to implement learning strategies that allow to develop these skills, on the basis that some skills are too complex and cannot be taught.

A training model from the perspective of the alliance would sensitize therapists in training on the verbal and nonverbal exchange that occurs between clients and therapists, how therapists respond to their clients and vice versa.

\section{Implications and cultural influences}

The effects of culture in the alliance and adherence should be considered for the interpretation of the results of any study. The sample consisted of families and therapists of upper-middle socioeconomic class, with a high level of education and living in an urban and industrialized area. The effects of culture on the therapeutic alliance and adherence is unclear and needs to explore the cultural differences between clients and therapists to understand how they affect the establishment of the alliance and adherence. The effects of culture on the therapeutic alliance and adherence are unclear and need to be explored taking into consideration the cultural differences between clients and therapists

\section{Research implications}

Future research must consider several limitations of this study. As it was mentioned previously, we need to carry more studies related to the adherence, therapeutic alliance and therapy outcome. Additionally, future studies might use a sample that provides greater gender and age balance with clients and therapists.

\section{References}

Basterra, M. (1999). El cumplimiento terapéutico. Pharmaceutical Care, 1(1), 97-106.

Beck, M., Friedlander, M.L., Escudero, V. (2006). Three perspectives on clients' experiences of the therapeutic alliance: a discovery-oriented investigation. Journal of Marital and Family Therapy, 32, 355-368.

Blow, A.J., \& Sprenkle, D. (2001). Common factors across theories of marriage and family therapy: A modified Delphi study. Journal of Marital and Family Therapy, 27, 385-392.

Bordin, E. (1979). The generalizability of the psychoanalytic concept of working alliance. Psychotherapy, Theory, Research and Practice, 16(3), 252-260.

Díaz, I. (2010). Construcción de un instrumento para medir la adherencia a la terapia familiar. Psicoterapia y Familia, 23(1), 53-61 
Di Mateo, R., Di Nicola, D. (1985). Achieving patient compliance. The psychology of the Medical Practitioner's role. New York, E.E.U.U: Pergamon.

Dinger, U., Strack, M., Leischsenring, F., Schauenberg, H. (2007). Influences of patients' and therapists' interpersonal problems and therapeutic alliance on outcome in psychotherapy. Psychotherapy Research, 17(2), 148-159.

Escudero, V., Friedlander, M.L., Varela,N., Abascal, A. (2008). Observing the therapeutic alliance in family therapy: associations with participants' perceptions and therapeutic outcomes. Journal of Family Therapy, 30, 194-214.

Horvath, A. (2006). The alliance in context: accomplishments, challenges and future directions. Psychotherapy: Theory, Research, Practice, Training,43, 292-299.

Johnson, L., Ketring, S. (2006). The therapy alliance: a moderator in therapy outcome for families dealing with child abuse and neglect. Journal of Marital and Family Therapy, 32 (3), 345-354.

Johnson, L., Wright, D. (2002). Revisiting Bordin's theory on the therapeutic alliance: implications for family therapy. Contemporary Family Therapy, 24(2), 257-269.

Knobloch-Fedders, L., Pinsof, W., Mann, B. (2004). The formation of the therapeutic alliance in couple therapy. Family Process, 43(4), 425-442.

Martín, L. (2004). Acerca del concepto de adherencia terapéutica. Revista Cubana de Salud Pública, 30 (4). Recuperado el 6 de noviembre de: http://scieloprueba.sld.cu/scielo.php?script=sci_artt ext\&pid=S086434662004000400008\&lng=\&nrm=is o\&tlng=

Pinsof, W. (1995). Integrative problem-centered therapy: a synthesis of family, individual, and biological therapies. New York, E.E.U.U: Harper Collins.

Rait, D. (2000). The therapeutic alliance in couples and family therapy. Psychotherapy in practice, 56(2), 211-224.

Sprenkle, D., Blow, A. (2004). Common factors and our sacred models. Journal of Marital and Family Therapy, 30, 113-129.

Stevens, C., Muran, C., Safran, J., Gorman, B., Winston, A. (2007). Levels and Patterns of the therapeutic alliance in brief psychotherapy. American Journal of Psychotherapy, 61(2), 109129.

\section{Declaration of Conflicting Interests}

The author has no conflicts of interest to report.

\section{Funding}

The author received no financial support for the research or the publication of this article. 